\title{
Effects of quadratic and cubic nonlinearities on a perfectly tuned parametric amplifier
}

\author{
Neumeyer, Stefan; Sorokin, Vladislav; Thomsen, Jon Juel
}

Published in:

Journal of Sound and Vibration

Link to article, DOI:

10.1016/j.jsv.2016.09.013

Publication date:

2016

Document Version

Peer reviewed version

Link back to DTU Orbit

Citation (APA):

Neumeyer, S., Sorokin, V., \& Thomsen, J. J. (2016). Effects of quadratic and cubic nonlinearities on a perfectly tuned parametric amplifier. Journal of Sound and Vibration, 386, 327-335.

https://doi.org/10.1016/j.jsv.2016.09.013

\section{General rights}

Copyright and moral rights for the publications made accessible in the public portal are retained by the authors and/or other copyright owners and it is a condition of accessing publications that users recognise and abide by the legal requirements associated with these rights.

- Users may download and print one copy of any publication from the public portal for the purpose of private study or research.

- You may not further distribute the material or use it for any profit-making activity or commercial gain

- You may freely distribute the URL identifying the publication in the public portal

If you believe that this document breaches copyright please contact us providing details, and we will remove access to the work immediately and investigate your claim 


\title{
Effects of quadratic and cubic nonlinearities on a perfectly tuned parametric amplifier
}

\author{
S. Neumeyer, V. S. Sorokin, J. J. Thomsen \\ Department of Mechanical Engineering, Solid Mechanics, \\ Technical University of Denmark, Denmark
}

\begin{abstract}
We consider the performance of a parametric amplifier with perfect tuning (two-to-one ratio between the parametric and direct excitation frequencies) and quadratic and cubic nonlinearities. A forced Duffing-Mathieu equation with appended quadratic nonlinearity is considered as the model system, and steady-state solutions and corresponding stabilities are obtained by the method of varying amplitudes. Some general effects of pure quadratic, and mixed quadratic and cubic nonlinearities on parametric amplification are shown. In particular, the effects of mixed quadratic and cubic nonlinearities may generate additional amplitude-frequency solutions. In this case an increased response and a more phase sensitive amplitude (phase between excitation frequencies) is obtained, as compared to the case with either pure quadratic or cubic nonlinearity. Furthermore, jumps and bi-stability in the amplitude-phase characteristics are predicted, supporting previously reported experimental observations.
\end{abstract}

Keywords: parametric amplification, quadratic and cubic nonlinearities, method of varying amplitudes

\section{Introduction}

Parametrically amplifying (adding parametric to direct excitation for boosting resonant oscillations) microelectromechanical resonators, which in recent years have been used for filtering and sensoring [1,2], can be advantageous for low-noise signal amplification [3], and appear promising for energy harvesting $[4,5]$. They can conveniently be modelled with an appended cubic nonlinearity [6], reflecting the symmetric effects of nonlinear curvature or 
midplane stretching [7], with the nonlinear effects being comparably stronger due to the small length scale [8]. The effects of pure cubic nonlinearity for a parametric amplifier have been investigated in [9].

The effect of mixed quadratic and cubic nonlinearities is considered in the present work for two reasons. First, the quadratic nonlinearity can conveniently be introduced alongside the cubic nonlinearity as a correction term of the mathematical model. In this way it appears in the governing equation of motion as small compared to the cubic term. Secondly, the study of relatively strong quadratic nonlinearity is also relevant because it can model an asymmetry in restoring forces of elastic structures [10, 11], e.g. due to buckling or initial curvature. The quadratic nonlinearity may even overcome the cubic nonlinearity, if the static deflection is large, or when the beam is very slender [12]. Therefore this study is motivated by an interest in general effects on parametric amplifiers, of both pure quadratic nonlinearity, and mixed quadratic and cubic nonlinearities.

Several works report on combined parametric and direct excitation including quadratic and cubic nonlinearities [13-15]. Commonly a perturbation method is applied, assuming damping, nonlinear, and excitation terms to be small, and that subthreshold (response dominated by the direct excitation component) pumping (adding parametric excitation) is applied. In some cases it is also assumed that the quadratic nonlinearity is smaller than the cubic nonlinearity, e.g. [16]. The present work considers both when the quadratic nonlinearity is smaller and larger than the cubic nonlinearity, and focuses on superthreshold (response dominated by the parametric excitation component) pumping. Superthreshold pumping is of interest because of the potentially higher achievable gains [9], as compared to being operated below their linear instability threshold, i.e. for subthreshold pumping.

In Sect. 2 a model system is proposed and the corresponding steadystate vibration amplitude is solved for using the method of varying amplitudes (MVA), for the cases of pure as well as mixed cubic and quadratic nonlinearities. In Sect. 3 these results are compared with results of direct numerical integration, showing good agreement. In Sect. 4 main conclusions of the paper are outlined. 


\section{Steady-state response analysis}

A forced Duffing-Mathieu equation with unit-normalized linear natural frequency and additional quadratic nonlinearity is investigated:

$$
\ddot{x}+\beta \dot{x}+(1+p \cos (2 \Omega t)) x+k_{2} x^{2}+k_{3} x^{3}=d \cos (\Omega t+\phi),
$$

where $\left({ }^{\circ}\right)$ denotes temporal derivatives, $\beta=2 \zeta$ where $\zeta$ is the damping ratio, $k_{2}$ is a quadratic nonlinearity coefficient, $k_{3}$ is a cubic nonlinearity coefficient, (...) $x$ describes the linear elastic restoring force, $p$ is a parametric excitation amplitude, $d$ is a direct excitation amplitude, $t$ is time, and $\phi$ is the phase between the external and parametric excitation. A similar system but without quadratic nonlinearity has been investigated in [9]. It is the simplest one degree of freedom system which captures the effects of linear damping, quadratic and cubic nonlinearities, and which has both direct and parametric excitation, which are necessary for a parametric amplifier. Such a system is also physically easy to realize approximately [17-20].

To solve the steady-state oscillations $x(t)$ of (1) approximately, the MVA is employed, as proposed in [21], where one assumes a harmonic series solution form:

$$
x(t)=\sum_{m=0}^{n} A_{m 1}(t) \cos (m \Omega t)+A_{m 2}(t) \sin (m \Omega t),
$$

where the amplitudes $A_{m 1}$ and $A_{m 2}$ are time-varying, and not required to vary slowly. This is contrary to the method of harmonic balance, where the coefficients $A_{m 1}$ and $A_{m 2}$ would be constants and (2) an approximation. The allowed time dependency of $A_{m 1}$ and $A_{m 2}$ means that (2) merely represents a shift of variables, by which the solution form (2) is exact for any value of $n$. The transition from $x$ to $2 n+1$ new variables, $A_{m 1}$ and $A_{m 2}\left(A_{02}=0\right)$, implies that a total of $2 n+1$ equations are needed. Inserting (2) into (1) and requiring the coefficients of the involved harmonic terms to vanish identically, we introduce $2 n$ additional equations; equation $2 n+1$ then includes the remaining harmonic terms, including those having order higher than $n$. Considering $n=2$ and thus the first three harmonics in (2), yields:

$$
\begin{aligned}
x(t)=A_{01}(t)+A_{11}(t) \cos (\Omega t)+ & A_{12}(t) \sin (\Omega t) \\
& +A_{21}(t) \cos (2 \Omega t)+A_{22}(t) \sin (2 \Omega t),
\end{aligned}
$$


and one obtains the following five equations with five variables $A_{11}, A_{12}, A_{21}$, $A_{22}$, and $A_{01}$ to solve for:

$$
\begin{gathered}
\ddot{A}_{11}+\beta \dot{A}_{11}+2 \Omega \dot{A}_{12}+\beta \Omega A_{12}+A_{11}\left(1-\Omega^{2}+\frac{1}{2} p+2 k_{2} A_{01}+3 k_{3} A_{01}^{2}\right) \\
+\left(3 k_{3} A_{01}+k_{2}\right)\left(A_{11} A_{21}+A_{12} A_{22}\right) \\
+\frac{3}{2} k_{3} A_{11}\left(\frac{1}{2}\left(A_{11}^{2}+A_{12}^{2}\right)+A_{21}^{2}+A_{22}^{2}\right)=d \cos (\phi), \quad(4) \\
\ddot{A}_{12}+\beta \dot{A}_{12}-2 \Omega \dot{A}_{11}-\beta \Omega A_{11}+A_{12}\left(1-\Omega^{2}-\frac{1}{2} p+2 k_{2} A_{01}+3 k_{3} A_{01}^{2}\right) \\
+\left(3 k_{3} A_{01}+k_{2}\right)\left(A_{11} A_{22}-A_{12} A_{21}\right) \\
+\frac{3}{2} k_{3} A_{12}\left(\frac{1}{2}\left(A_{11}^{2}+A_{12}^{2}\right)+A_{21}^{2}+A_{22}^{2}\right)=-d \sin (\phi), \quad(5) \\
+\frac{1}{2} k_{2}\left(A_{11}^{2}-A_{21}^{2}+4 A_{01} A_{21}\right)+3 k_{3} A_{21}\left(\frac{1}{4}\left(A_{21}^{2}+A_{22}^{2}\right)+\frac{1}{2}\left(A_{11}^{2}+A_{12}^{2}\right)+A_{01}^{2}\right) \\
\quad+\frac{3}{2} k_{3} A_{01}\left(A_{11}^{2}-A_{12}^{2}\right)=0, \quad(6) \\
\ddot{A}_{21}+\beta \dot{A}_{21}+4 \Omega A_{21}+A_{21}+p A_{01}+2 \Omega\left(\beta A_{22}-2 \Omega A_{21}\right) \\
\ddot{A}_{22}+\beta \dot{A}_{22}-4 \Omega \dot{A}_{21}-2 \beta \Omega A_{21} \\
+A_{11} A_{12}\left(k_{2}+3 k_{3} A_{01}\right)+A_{22}\left(1-4 \Omega^{2}+2 k_{2} A_{01}\right) \\
+3 k_{3} A_{22}\left(\frac{1}{4}\left(A_{21}^{2}+A_{22}^{2}\right)+\frac{1}{2}\left(A_{11}^{2}+A_{12}^{2}\right)+A_{01}^{2}\right)=0, \quad(7)
\end{gathered}
$$




$$
\begin{gathered}
\ddot{A}_{01}+\beta \dot{A}_{01}+A_{01}+\frac{1}{2} p A_{21}+\frac{3}{2} k_{3} A_{11} A_{12} A_{22} \\
+\frac{3}{4} k_{3} A_{21}\left(A_{11}^{2}-A_{12}^{2}\right)+k_{2}\left(\frac{1}{2}\left(A_{11}^{2}+A_{21}^{2}+A_{22}^{2}\right)+A_{01}^{2}\right) \\
+k_{3} A_{01}\left(\frac{3}{2}\left(A_{11}^{2}+A_{12}^{2}+A_{21}^{2}+A_{22}^{2}\right)+A_{01}^{2}\right)= \\
-\frac{1}{4}\left(\left\{k_{3} A_{11}^{3}-2 A_{12}\left(2 k_{2}+6 k_{3} A_{01}-3 k_{3} A_{21}\right) A_{22}\right.\right. \\
\left.+A_{11}\left[2\left(p+2 k_{2} A_{21}\right)-3 k_{3}\left(A_{12}^{2}-A_{21}\left(4 A_{01}+A_{21}\right)+A_{22}^{2}\right)\right]\right\} \cos (3 \Omega t) \\
+\left\{A_{21}\left(3 k_{3} A_{11}^{2}-3 k_{3} A_{12}^{2}+2\left[p+\left(k_{2}+3 k_{3} A_{01}\right) A_{21}\right]\right)\right. \\
\left.-6 k_{3} A_{11} A_{12} A_{22}-2\left(k_{2}+3 k_{3} A_{01}\right) A_{22}^{2}\right\} \cos (4 \Omega t) \\
+3 k_{3}\left\{-2 A_{12} A_{21} A_{22}+A_{11}\left(A_{21}^{2}-A_{22}^{2}\right)\right\} \cos (5 \Omega t) \\
+k_{3} A_{21}\left\{A_{21}^{2}-3 A_{22}^{2}\right\} \cos (6 \Omega t) \\
+\left\{-k_{3} A_{12}^{3}+2 A_{11}\left(2 k_{2}+6 k_{3} A_{01}+3 k_{3} A_{21}\right) A_{22}+A_{12}\left[2\left(p+2 k_{2} A_{21}\right)\right.\right. \\
\left.\left.+3 k_{3}\left(A_{11}^{2}+4 A_{01} A_{21}-A_{21}^{2}+A_{22}^{2}\right)\right]\right\} \sin (3 \Omega t) \\
+\left\{6 k_{3} A_{11} A_{12} A_{21}+\left[2 p+3 k_{3} A_{11}^{2}-3 k_{3} A_{12}^{2}+4\left(k_{2}+3 k_{3} A_{01}\right) A_{21}\right] A_{22}\right\} \sin (4 \Omega t) \\
+3 k_{3}\left\{2 A_{11} A_{21} A_{22}+A_{12}\left(A_{21}^{2}-A_{22}^{2}\right)\right\} \sin (5 \Omega t) \\
\left.-k_{3} A_{22}\left\{-3 A_{21}^{2}+A_{22}^{2}\right\} \sin (6 \Omega t)\right) . \quad(8)
\end{gathered}
$$

Eqs. (4)-(8) cannot be solved exactly. To obtain approximate solutions we neglect higher $(>n)$ harmonics in $(8)$, i.e. all of the right-hand side, so that:

$$
\begin{aligned}
& \ddot{A}_{01}+\beta \dot{A}_{01}+A_{01}+ \\
&+\frac{1}{2} p A_{21}+\frac{3}{2} k_{3} A_{11} A_{12} A_{22} \\
&+\frac{3}{4} k_{3} A_{21}\left(A_{11}^{2}-A_{12}^{2}\right)+k_{2}\left(\frac{1}{2}\left(A_{11}^{2}+A_{21}^{2}+A_{22}^{2}\right)+A_{01}^{2}\right) \\
& \quad+k_{3} A_{01}\left(\frac{3}{2}\left(A_{11}^{2}+A_{12}^{2}+A_{21}^{2}+A_{22}^{2}\right)+A_{01}^{2}\right)=0 .
\end{aligned}
$$

This simplification is adequate when in (1) the quadratic nonlinearity term $k_{2} x^{2}$, the cubic nonlinearity term $k_{3} x^{3}$, and the linear parametric excitation term $p x$ are small compared to the linear restoring term $x$. Steady-state solutions are obtained by requiring all time derivatives in (4)-(7), (9) to vanish, so that:

$$
\begin{aligned}
\beta \Omega A_{12}+A_{11}(1- & \left.\Omega^{2}+\frac{1}{2} p+2 k_{2} A_{01}+3 k_{3} A_{01}^{2}\right) \\
& +\left(3 k_{3} A_{01}+k_{2}\right)\left(A_{11} A_{21}+A_{12} A_{22}\right) \\
& +\frac{3}{2} k_{3} A_{11}\left(\frac{1}{2}\left(A_{11}^{2}+A_{12}^{2}\right)+A_{21}^{2}+A_{22}^{2}\right)=d \cos (\phi),
\end{aligned}
$$




$$
\begin{aligned}
& -\beta \Omega A_{11}+A_{12}\left(1-\Omega^{2}-\frac{1}{2} p+2 k_{2} A_{01}+3 k_{3} A_{01}^{2}\right) \\
& +\left(3 k_{3} A_{01}+k_{2}\right)\left(A_{11} A_{22}-A_{12} A_{21}\right) \\
& +\frac{3}{2} k_{3} A_{12}\left(\frac{1}{2}\left(A_{11}^{2}+A_{12}^{2}\right)+A_{21}^{2}+A_{22}^{2}\right)=-d \sin (\phi), \\
& A_{21}+p A_{01}+2 \Omega\left(\beta A_{22}-2 \Omega A_{21}\right) \\
& +\frac{1}{2} k_{2}\left(A_{11}^{2}-A_{21}^{2}+4 A_{01} A_{21}\right)+3 k_{3} A_{21}\left(\frac{1}{4}\left(A_{21}^{2}+A_{22}^{2}\right)+\frac{1}{2}\left(A_{11}^{2}+A_{12}^{2}\right)+A_{01}^{2}\right) \\
& +\frac{3}{2} k_{3} A_{01}\left(A_{11}^{2}-A_{12}^{2}\right)=0 \text {, } \\
& -2 \beta \Omega A_{21}+A_{11} A_{12}\left(k_{2}+3 k_{3} A_{01}\right)+A_{22}\left(1-4 \Omega^{2}+2 k_{2} A_{01}\right) \\
& +3 k_{3} A_{22}\left(\frac{1}{4}\left(A_{21}^{2}+A_{22}^{2}\right)+\frac{1}{2}\left(A_{11}^{2}+A_{12}^{2}\right)+A_{01}^{2}\right)=0 \text {, } \\
& A_{01}+\frac{1}{2} p A_{21}+\frac{3}{2} k_{3} A_{11} A_{12} A_{22} \\
& +\frac{3}{4} k_{3} A_{21}\left(A_{11}^{2}-A_{12}^{2}\right)+k_{2}\left(\frac{1}{2}\left(A_{11}^{2}+A_{21}^{2}+A_{22}^{2}\right)+A_{01}^{2}\right) \\
& +k_{3} A_{01}\left(\frac{3}{2}\left(A_{11}^{2}+A_{12}^{2}+A_{21}^{2}+A_{22}^{2}\right)+A_{01}^{2}\right)=0,
\end{aligned}
$$

from which the steady-state amplitudes $A_{11}, A_{12}, A_{21}, A_{22}$, and $A_{01}$ can be determined. Note that considering $n=1$ in (2) is sufficient for pure cubic nonlinearity, in order to obtain a good agreement between the approximate analytical results, and the corresponding results obtained by direct numerical integration. In the case of pure quadratic nonlinearity, however, one needs at least $n=2$, otherwise the softening effect will remain a part of the higher $(>n)$ harmonics, which is neglected in (9).

Since $A_{m 1}$ and $A_{m 2}$ for $m>0$ are amplitudes of the time harmonic response (cf. (2)), eqs. (10)-(14) can be used to calculate frequency responses, and thus to assess the effects of quadratic and cubic nonlinearities on a perfectly tuned parametric amplifier. The amplitudes $A_{m 1}$ and $A_{m 2}$ are determined from (10)-(14) approximately by neglecting nonlinearities in $A_{21}, A_{22}$, and $A_{01}$. The approximate analytical steady-state amplitude $a$ is also here computed as the absolute maximum of $x(t)$ (cf. (2)). Backbones are then derived by zeroing the forcing and damping terms. The steadystate amplitude-frequency solutions could also have been obtained using the method of Harmonic Balance [22]. However, the MVA also provides the modulation equations (4)-(7), (9) from which stability of the steady-state solutions can be determined by evaluating Jacobian eigenvalues. The response $x(t)$ is also found by direct numerical integration of $(1)$, and the corresponding steady-state amplitude is computed as the absolute maximum of $x(t)$. 


\section{Results}

Fig. 1 shows various steady-state amplitude-frequency relations for suband superthreshold pumping in the case of no nonlinearity, pure cubic nonlinearity, pure quadratic nonlinearity, and mixed cubic and quadratic nonlinearities. Fig. (a) shows that classical responses with softening or hardening
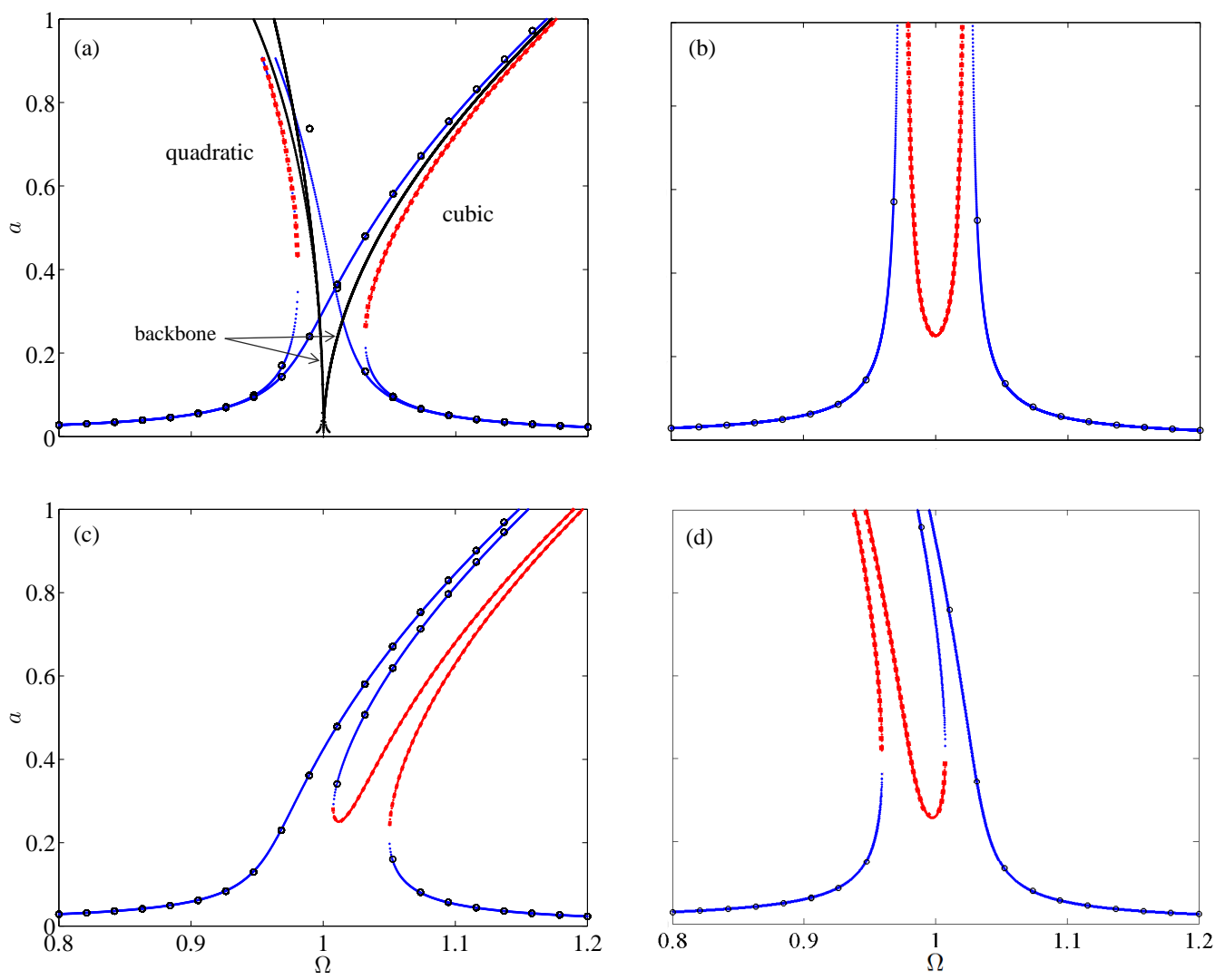

Figure 1: Steady-state amplitude-frequency relations showing (a) subthreshold ( $p=0.01)$ quadratic $\left(k_{2}=0.3, k_{3}=0\right)$ and cubic $\left(k_{2}=0, k_{3}=0.5\right)$ responses, and their respective backbones (b) linear $\left(k_{2}=k_{3}=0\right)$ superthreshold $(p=0.1)$ response (c) pure cubic $\left(k_{2}=\right.$ $\left.0, k_{3}=0.3\right)$ superthreshold response (d) pure quadratic $\left(k_{2}=0.3, k_{3}=0\right)$ superthreshold response. Solid blue curve and solid red curve with squares respectively denote stable and unstable approximate analytical results. Solid black curves denote backbones. Circles denote results obtained by direct numerical integration of $(1) . \quad(\phi=-\pi / 4, \beta=0.01$, $d=0.01)$.

are obtained for subthreshold pumping. In the case of pure quadratic nonlinearity, the backbone to the left is obtained by direct numerical integration, 
whereas the one to the right is obtained using the MVA. The discrepancy for the latter is due to neglecting nonlinearities in amplitudes $A_{21}, A_{22}$, and $A_{01}$, as mentioned in Sect. 2. Note also that for the backbones, the amplitude equals zero at the linear natural frequency and approaches infinity in the linear case, as would be expected. Fig. (b) depicts linear superthreshold pumping. As compared to the subthreshold case, an extra peak emerges, and the solutions are now dominated by the parametric excitation component. Fig. (c) shows superthreshold pumping with pure cubic nonlinearity. Contrary to the classical hardened Duffing response where the lower solution on the peak is unstable, here the lower solution on the upper peak is stable. This observation has been reported previously [9]. Fig. (d) shows superthreshold pumping with pure quadratic nonlinearity. The response is similar to that of negative cubic nonlinearity (softening) and opposite of positive cubic nonlinearity (hardening). As in the case with pure cubic nonlinearity, the shift from sub- to superthreshold pumping results in the emergence of an extra peak, and a partial shift in stability of the solutions. A good agreement between results of direct numerical simulation and approximate analytical results is obtained in all of the above cases. Results of superthreshold pumping with cubic nonlinearity and a quadratic nonlinearity correction term are not shown in the figures; the otherwise hardened response, due to a positive cubic nonlinearity, simply becomes less hardened. This is similar to the case for subthreshold pumping.

Fig. 2 presents the response for mixed quadratic and cubic nonlinearities for sub- and superthreshold pumping where, respectively, below and above unit amplitude the quadratic and cubic nonlinearities dominate the response. The results in fig. (a) for subthreshold pumping have been reported elsewhere [15], except than in our case the unstable solution on the upper part of the peak is too close to the stable solution to be visible. The insert shows that the approximate analytical response $x(t)$ as given by (2), of which the absolute maximum value is chosen as the amplitude $a$, is slightly anharmonic lacking symmetry with respect to $x=0$. That is, even harmonics are present, but it is still reasonable to choose the absolute maximum value of the response $x(t)$ as the amplitude $a$, since the approximate analytical response and the response obtained through direct numerical integration are similar. For superthreshold pumping (fig. (b)) the response becomes more intricate: Two additional solutions emerge, compared to the case of superthreshold pumping with pure cubic nonlinearity in which a maximum of five solutions coexist (Fig. 1(c)), so that a maximum of seven solutions coexist, e.g. for $\Omega=1$. 

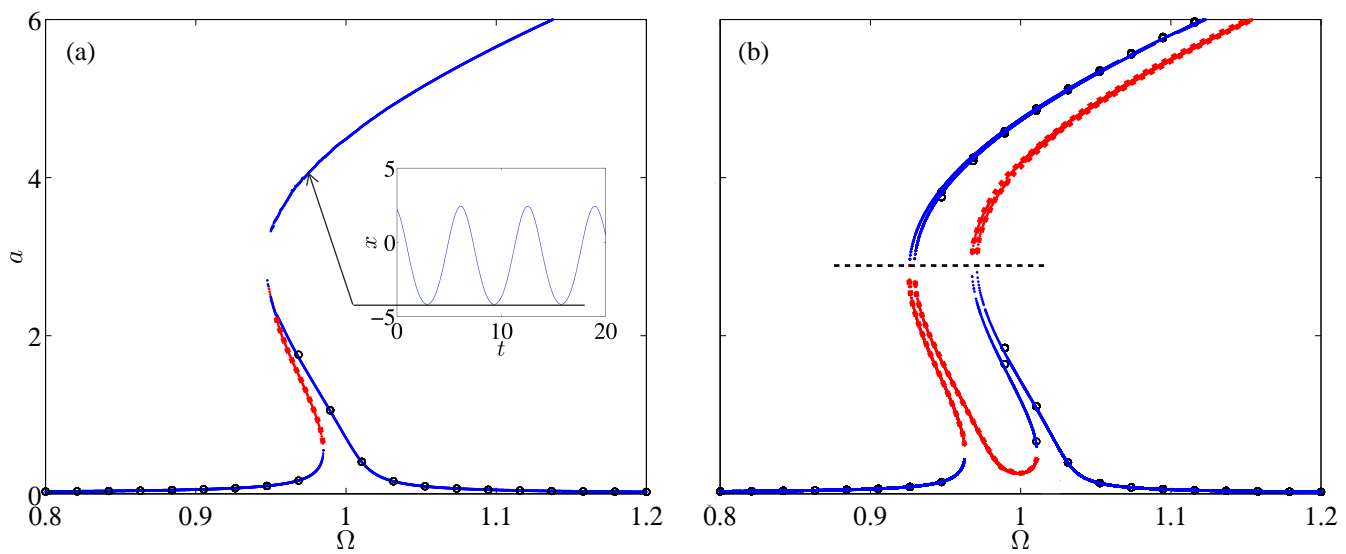

Figure 2: Steady-state amplitude-frequency relations with mixed quadratic and cubic nonlinearities $\left(k_{2}=0.3, k_{3}=0.05\right)$ for (a) sub- and (b) superthreshold pumping. Parameter values and markers as used in Fig. 1 unless stated otherwise.

For lower amplitudes (below the dashed line in fig. (b)), the stability of the peaks is similar to the case of pure quadratic nonlinearity (Fig. 1(d)), and for higher amplitudes similar to the case of pure cubic nonlinearity (Fig. 1(c)). Even though the amplitude is large and the analytical solution is approximate, it is still validated by results of direct numerical integration.

Fig. 3 depicts the case where the effects of quadratic and cubic nonlinearities cancel out for amplitudes smaller than unity, i.e. a close to linear response is obtained for an extended range of amplitudes as compared to when the effects of quadratic and cubic nonlinearities do not cancel out. Fig. (a) shows the response for subthreshold pumping whereas fig. (b) shows the response for superthreshold pumping. The insert in fig. (a) shows that the response $x(t)$ is almost symmetric with respect to $x=0$. Significantly larger amplitudes than for pure quadratic or cubic nonlinearity (figs. 1(c,d)), are obtained in both cases. This could be useful for e.g. sensor applications and energy harvesters utilizing parametric amplification. Besides the benefits of tuning the device such that a higher steady-state amplitude is realized, the obtained response of a parametric amplifier is also of general interest, since one may encounter systems where the effect of quadratic nonlinearity cancels that of the cubic nonlinearity, or even becomes dominant [10-12], as also mentioned in the introduction. Note that the stability of the solutions is different than for the linear case (Fig. 1(b)).

Fig. 4 presents steady-state amplitude-frequency relations for paramet- 

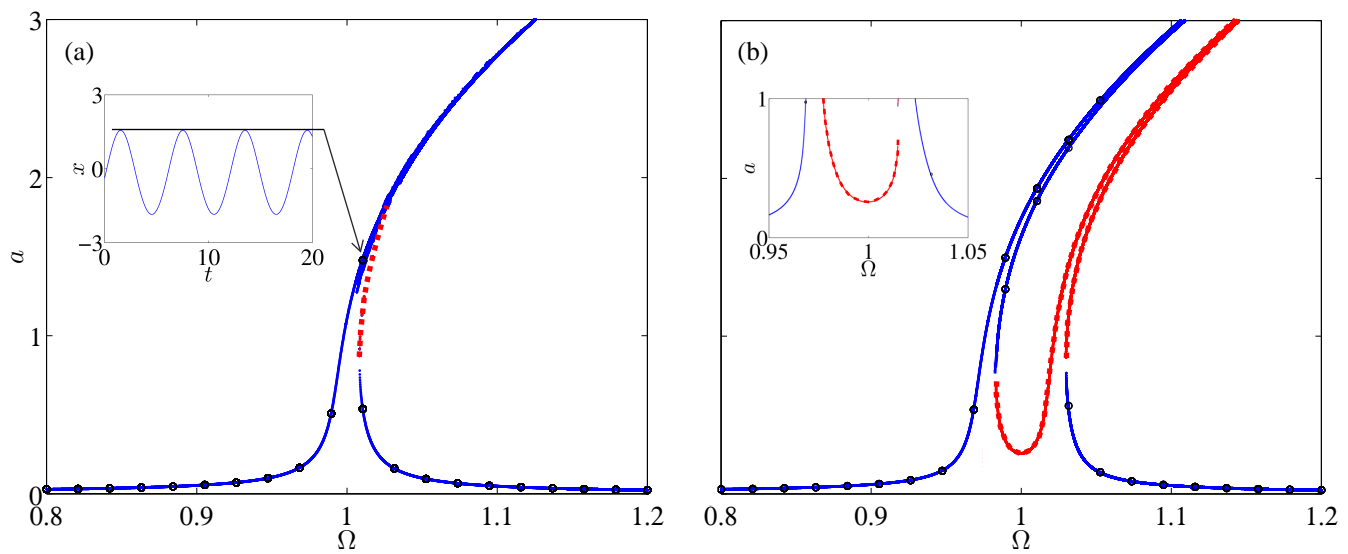

Figure 3: Steady-state amplitude-frequency relations where the effects of quadratic and cubic nonlinearities cancel out for $a<1\left(k_{2}=0.3, k_{3}=0.1\right)$ for (a) sub- and (b) superthreshold pumping where the insert in (a) shows the response $x(t)$ and the insert in (b) is a blow up. Parameter values and markers as used in Fig. 1 unless stated otherwise.

ric excitation $p$ ranging from sub- to superthreshold pumping. The response for $p \simeq 0$ in each subfigure is for subthreshold pumping, whereas the two other responses in each figure are for superthreshold pumping, as can also be seen by the emergence of additional solutions. Fig. (a) shows the linear case. The minimum of the unstable response, i.e. the response at $\Omega \approx 1$, lowers with increasing pumping, and the frequency separation between the peaks on each response curve increases with increasing pumping. Figs. (b-d) show, respectively, in the case of pure cubic nonlinearity, pure quadratic nonlinearity, and for mixed cubic and quadratic nonlinearities, a transition from sub- to superthreshold pumping where the peak separation increase with increased pumping, as in the linear case.

Fig. 5 shows steady-state subthreshold amplitude-phase relations for pure quadratic, pure cubic, and various combinations of mixed quadratic and cubic nonlinearities. Fig. (a) reveals that for almost any phase $\phi$ the largest response is obtained in the linear case. Nonlinear effects reduce the amplitude, both in the case of pure quadratic nonlinearities, pure cubic nonlinearities, or mixed quadratic and cubic nonlinearities. Fig. (b) depicts a case where the effects of quadratic and cubic nonlinearities cancel near $\Omega \approx 1$. A significant increase in response is obtained; the maximum magnitude of the response is almost the same as in the linear case. But at the same time the amplitude-phase response differs qualitatively from the linear one; jumps and 

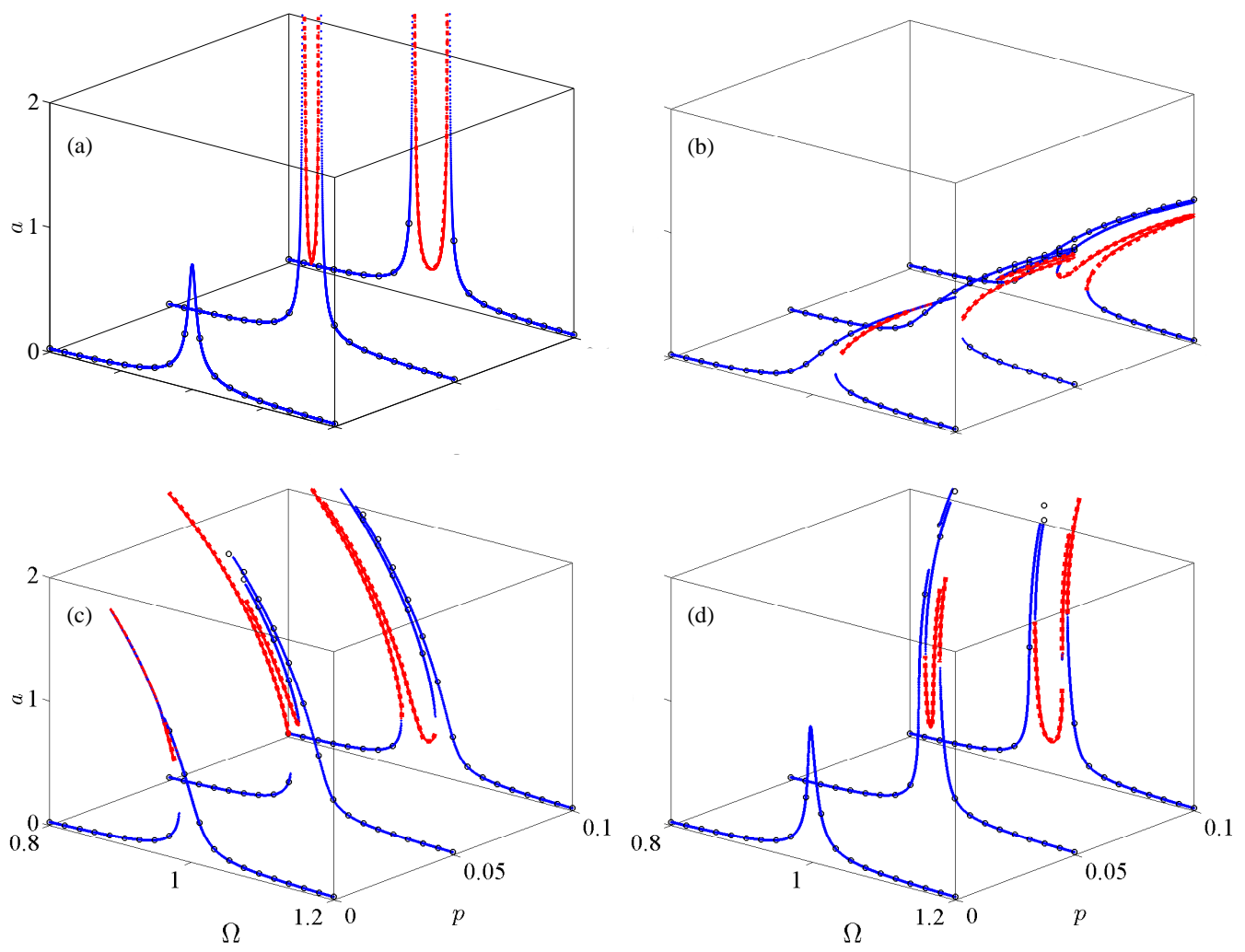

Figure 4: Steady-state amplitude-frequency relations for parametric excitation amplitude for (a) linear $\left(k_{2}=k_{3}=0\right)$ (b) pure cubic nonlinearity $\left(k_{2}=0, k_{3}=0.5\right)$ (c) pure quadratic nonlinearity $\left(k_{2}=0.3, k_{3}=0\right)(\mathrm{d})$ quadratic and cubic nonlinearities $\left(k_{2}=0.3\right.$, $\left.k_{3}=0.08\right)$. Parameter values and markers as used in Fig. 1 unless stated otherwise.

bi-stability are observed, similar to experimental results [23]. In all cases a good agreement between analytical approximate results and results of direct numerical integration is obtained. In general it is observed that with increasing nonlinear coefficients, the amplitude becomes less phase sensitive, except in the case where the effects of quadratic and cubic nonlinearities cancel out; here the amplitude can be even as sensitive as in the linear case. Steady-state superthreshold amplitude-phase relations are not shown since superthreshold parametric pumping appears to yield a phase-insensitive response. 

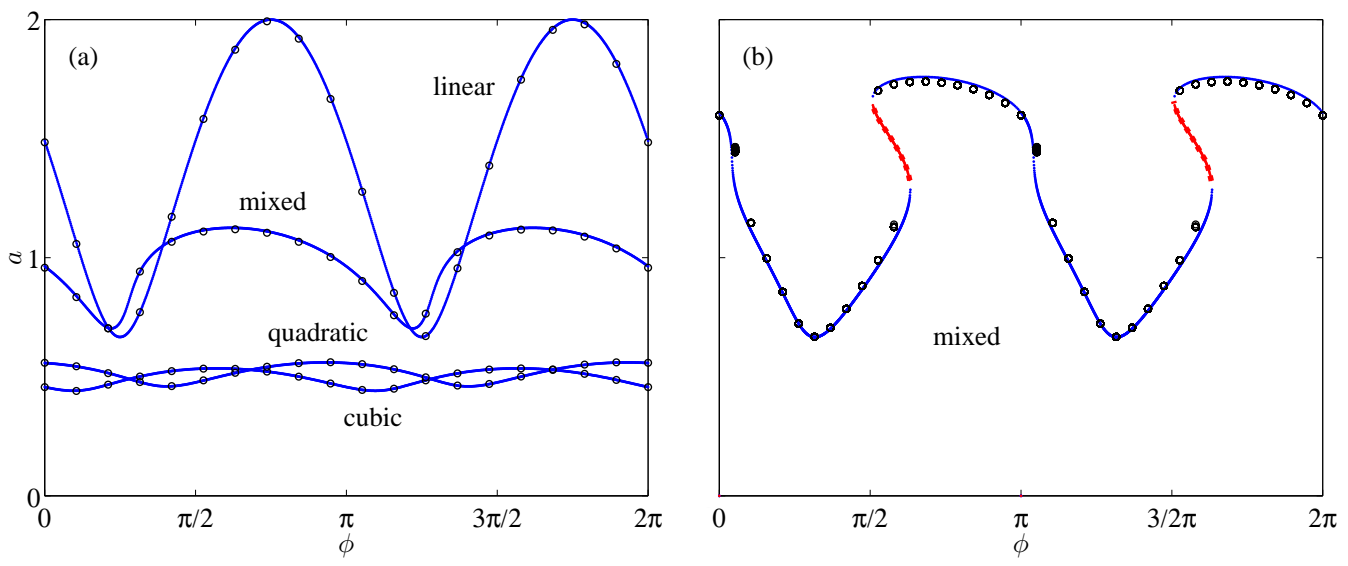

Figure 5: Steady-state subthreshold amplitude-phase relations for (a) linear $\left(k_{2}=k_{3}=0\right)$, pure quadratic $\left(k_{2}=0.3, k_{3}=0\right)$, pure cubic $\left(k_{2}=0, k_{3}=0.1\right)$, and mixed quadratic and cubic nonlinearities $\left(k_{2}=0.3, k_{3}=0.1\right)$ (b) mixed quadratic and cubic nonlinearities $\left(k_{2}=0.3, k_{3}=0.08\right)$. $(\Omega=1$.) Parameter values and markers as used in Fig. 1 unless stated otherwise.

\section{Conclusions}

The effects of quadratic and cubic nonlinearities on the response of a parametric amplifier were investigated. These effects are often inherently present for real amplifiers, but can also be purposefully introduced and utilized. Approximate analytical steady-state responses were obtained by the method of varying amplitudes and compared to results of direct numerical integration, showing good agreement. Mixed quadratic and cubic nonlinearities may generate additional amplitude-frequency solutions. When the effects of quadratic and cubic nonlinearities cancel out, a significantly increased response with a more phase sensitive amplitude is obtained, as compared to the case with pure quadratic or cubic nonlinearity. Jumps and bi-stability in the amplitude-phase characteristics were predicted theoretically, supporting previously reported experimental findings.

[1] R. Lifshitz, M. C. Cross, Nonlinear Dynamics of Nanomechanical and Micromechanical Resonators, Wiley-VCH, 2009.

[2] J. F. Rhoads, S. W. Shaw, K. L. Turner, Nonlinear dynamics and its applications in micro- and nanoresonators, Journal of Dynamic Systems, Measurement, and Control 132 (2010) 034001. 
[3] D. Rugar, P. Grütter, Mechanical parametric amplification and thermomechanical noise squeezing, Physical Review Letters 67 (1991) 699-702.

[4] M. F. Daqaq, D. Bode, Exploring the parametric amplification phenomenon for energy harvesting, Proceedings of the Institution of Mechanical Engineers, Part I: Journal of Systems and Control Engineering 225 (2011) 456-466.

[5] J. Eom, J. Lee, H. Lee, B. Choi, Energy harvesting for bladder pressure sensor using parametric amplification phenomenon of pvdf bimorph cantilever, in: Proceedings of $6^{\text {th }}$ Biomedical Engineering International Conference, BMEiCON, Krabi, Thailand, 2013, pp. 1-3.

[6] W. Zhang, R. Baskaran, K. Turner, Tuning the dynamic behavior of parametric resonance in a micromechanical oscillator, Applied Physics Letters 82 (2003) 130132.

[7] A. H. Nayfeh, D. T. Mook, Nonlinear Oscillations, Wiley, 1979.

[8] M. L. Roukes, Nanoelectromechanical Systems, 2000.

[9] J. F. Rhoads, S. W. Shaw, The impact of nonlinearity on degenerate parametric amplifiers, Applied Physics Letters 96 (2010) 234101.

[10] I. Kozinsky, H. C. Postma, I. Bargatin, M. Roukes, Tuning nonlinearity, dynamic range, and frequency of nanomechanical resonators, Applied Physics Letters 88 (2006) 253101.

[11] J. J. Thomsen, Vibrations and Stability: Advanced Theory, Analysis, and Tools, Springer, 2003.

[12] G. C. Hughes, C. W. Bert, Effect of gravity on nonlinear oscillations of a horizontal, immovable-end beam, Nonlinear Dynamics 3 (1992) 365-373.

[13] W. Zhang, G. Meng, Nonlinear dynamical system of micro-cantilever under combined parametric and forcing excitations in mems, in: Proceedings of $30^{\text {th }}$ annual conference of IEEE Industrial Electronics Society (IECON), Busan, South Korea, 2004, pp. $1571-1576$.

[14] Y. Amer, H. Bauomy, M. Sayed, Vibration suppression in a twin-tail system to parametric and external excitations, Computers and Mathematics with Applications 58 (2009) 1947.

[15] A. M. Elshurafa, K. Khirallah, H. H. Tawfik, A. Emira, A. K. S. A. Aziz, S. M. Sedky, Nonlinear dynamics of spring softening and hardening in folded-mems comb drive resonators, Journal of Microelectromechanical Systems 20 (2011) 943-958.

[16] G. S. Agnes, D. J. Inman, Nonlinear piezoelectric vibration absorbers, Smart materials and structures 5 (1996) 704. 
[17] J. F. Rhoads, N. J. Miller, S. W. Shaw, B. F. Feeny, Mechanical domain parametric amplification, Journal of Vibration and Acoustics 130 (2008) 061006.17.

[18] V. Kumar, J. K. Miller, J. F. Rhoads, Nonlinear parametric amplification and attenuation in a base-excited cantilever beam, Journal of Sound and Vibration 330 (2011) $5401-5409$.

[19] S. Neumeyer, J. J. Thomsen, Macroscale mechanical domain parametric amplification: superthreshold pumping and optimal excitation parameters, in: Proceedings of Time-Periodic Systems: Current trends in theory and application, EUROMECH Colloquium 532, Darmstadt, Germany, 2012, pp. 63-66.

[20] S. Neumeyer, J. J. Thomsen, Macromechanical parametric amplification with a baseexcited doubly clamped beam, in: Proceedings of $11^{\text {th }}$ International Conference on Vibration Problems, ICOVP 2013, Lisbon, Portugal, 2013. 2pp.

[21] V. S. Sorokin, J. J. Thomsen, Vibration suppression for strings with distributed loading using spatial cross-section modulation, Journal of Sound and Vibration 335 (2015) 66-77.

[22] V. V. Bolotin, The Dynamic Stability of Elastic Systems, Holden-Day, 1964.

[23] S. Neumeyer, R. V. D. Looij, J. J. Thomsen, Jumps and bi-stability in the phase-gain characteristics of a nonlinear parametric amplifier, in: Proceedings of $8^{\text {th }}$ European Nonlinear Dynamics Conference, ENOC 2014, Vienna, Austria, 2014. 2pp. 